\title{
Maximizing Quality Data-collection in Mobile-sink Based Energy-harvesting Sensor Networks
}

\author{
Naween Kumar, Dinesh Dash
}

\begin{abstract}
In energy-harvesting wireless sensor network (EHWSNs), sensor nodes (SNs) are rechargeable. But, the harvesting techniques used for recharging SNs' are dynamic and may not provide evenly harvested energy to all sensors. Thus, maximizing quality data-collection (MQDC) is an interesting issue under energy harvesting constraints. In this article, we focus on this issue to solve. We consider a mobile-sink (MS) that moves on a constrained-path for collecting SNs' data periodically. The SNs are distributed nearby the constrained-path. This scenario may exist in various real-world applications, such as traffic monitoring, environment monitoring and health monitoring of large buildings or bridges, etc. We transform the MQDC problem into a network-utility maximization problem. We then prove that the converted problem is an NP-hard. Thereafter, we develop a heuristic algorithm, referred to as Maximizing Quality Datacollection using Constrained-path Mobile-Sink (MQDCPMS), to solve it. We address the effect of change in the speed of MS on the quality data-collection. Finally, through extensive simulations, we find that the MQDCPMS algorithm maximizes the quality data-collection comparatively better than other baseline algorithms.
\end{abstract}

Index Terms: EH-WSNs, Quality data-collection, Mobile-sink, Utility maximization, Heuristic algorithm.

\section{INTRODUCTION}

Recently, a wireless sensor network is emerging as a prominent technology for various real-life applications, for instance, intelligent traffic monitoring [1], forest fire monitoring [2], and environmental monitoring, etc. [3]. The network comprised of limited battery-powered sensor nodes $(S N S)$ and a base- station $(B S)$. The $S N s$ sense data from their environment which might not be feasible for the large-scale WSNs. One of the possible solutions is the replacing of SNs' batteries. However, in some critical application environments, replacing $S N s^{\prime}$ batteries is a costly affair and sometimes may become impossible. In order to make the operations uninterrupted [4]-[6], another solution is the use of rechargeable SNs in the network, where the SNs harvest energy from their external environment such as wind system and solar panel system, etc. However, the dynamic characteristics of harvesting technique in energy-harvesting sensor networks (EH-WSNs), poses major challenges in the

Revised Manuscript Received on July 10, 2019.

Naween Kumar, Dept. of Computer Science \& Engineering, National Institute of Technology Patna, Patna, India.

Dinesh Dash, Dept. of Computer Science \& Engineering, National Institute of Technology Patna, Patna, India. quality data collection for such networks [5], [7].

In conventional WSNs, data collection technique is sometimes non-reliable as the network connectivity is not guaranteed. In addition, the $S N s$ nearby the $B S$ (base-station) have to relay more data traffic due to multi-hop transmission. Thus, they may be failed eventually due to the rapid depletion of their energy.

To mitigate these issues, a mobile-sink $(M S)$ based mobility is introduced for data collection [8] in EH-WSNs. Existing studies have shown that the exploitation of $M S$ for collecting sensed-data can improve the performances of the network such as data throughput [9], [10], and quality [11], [12]. However, due to the dynamics in energy harvesting, the WSNs researchers have been focusing on quality data collection for improving the performances of EH-WSNs [13], [14]. Therefore, maximizing quality data collection has been a key problem [15] in EH-WSNs, as the increment in network lifetime, is not an issue in such networks [12], [16].

For an application example, in Fig. 1, sensors are distributed nearby the trajectory-path $P_{m s}$ of $M S$ to sense data. The $M S$ moves on the $P_{m s}$ at a constant speed for collecting data from sensors via a single-hop relay. The objective is maximizing the quality data-collection from the directly reachable sensors. As a more quality data collection implies a more accurate estimation.

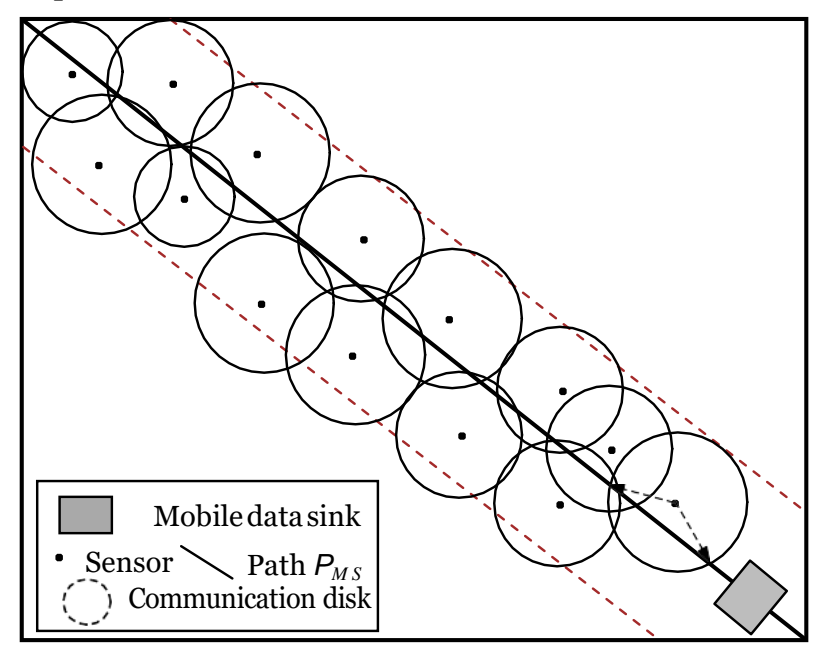

Fig 1. An example of Energy-harvesting wireless sensor network.

The major contributions are therefore summarized as follows: 


\section{Maximizing Quality Data-collection in Mobile-sink Based Energy-harvesting Sensor Networks}

\section{1) Propose a quality data-collection $(M Q D C)$ problem} using a constrained-path $M S$ with a fixed-speed.

2) Propose a heuristic algorithm referred to as Maximizing Quality Data-collection using constrained path Mobile- Sink (MQDCPMS).

The rest parts of our work are organized as follows: the related works of data-collection problems using $M S$ in EHWSNs are surveyed in section II. In section III, the network model, the proposed problem formulation and its NP-Hardness is presented. Section IV explains the solution of MQDCPMS algorithm and its run-time complexity. Section V presents the implementation-detail of MQDCPMS and its performances by simulation experiments. Finally, section VI infers our proposed work. Recently, various studies have been investigated on different kinds of data gathering techniques in EH-WSNs using mobile-sink(s) (MSs) [9], [16]-[18]. They have shown that they can improve the performances of the network. In [19], a linkscheduling optimization model is developed to maximize the data gathering in EH-WSN. The author's et al. in [19] exploited star topology for the communication network and proposed a super-frame structure for the base station to collect data. However, sensors used in the network are mobile. In our work, sensors are statics.

\section{RELATED WORKS}

Recently, various studies have been investigated on different kinds of data gathering techniques in EH-WSNs using mobile- sink(s) (MSs) [9], [16]-[18]. They have shown that they can improve the performances of the network. In [19], a link- scheduling optimization model is developed to maximize the data gathering in EH-WSN. The author's et al. In [19] exploited star topology for the communication network and proposed a super-frame structure for the base station to collect data. However, sensors used in the network are mobile. In our work, sensors are statics.

In [17], a set of sensors and some sojourn (stop) locations are deployed in the network. There is a controllable-path mobile-sink $M S$ which collects sensed-data from sensors in SNs by staying at the sojourn locations. The authors et al. in [17] developed a heuristic algorithm for finding a tour of $M S$ and the scheduling time of the sojourn locations, such that data throughput of the network is maximized. But, the data-throughpu $\mathrm{t}$ improved by the algorithm is not significant. In our work, the path of $M S$ is constrained not controllable. In [16], Ren et al. proposed a maximizing data-collection problem in EH-WSNs using constrained-path mobile-sink. They first transformed the maximizing data-collection problem into a generalized assignment problem and then solved it by developing a better approximation algorithm. Similar to this problem, the authors in [9], [18] eliminated the constraints of fixed time-slot period and common communication range for maximizing the data-collection of the network. They converted their problems into nonlinear programming (NLP) problems by considering the acceleration and deceleration time duration and the data delivery latency.
However, in this work, the amount of data-collection is maximized, which is different from quality data maximization. In our proposed work, we are maximizing the quality data collection in EH-WSNs. In [13], a distributed algorithm is proposed for maximizing the quality data collection in EH-WSNs. They first determined anchor points among sensors based on the sensor's energy level and data availabilities and given delay time. However, in their work, multi-hop communication relay is used for data forwarding among sensors and $M S$ and the trajectory-path of $M S$ is controllable, on the other hand, in our work, the trajectory-path of $M S$ is fixed.

In [11], [12], the $M S$ moves along a fixed trajectory-path for collecting sensed-data through single-hop periodically in an EH-WSN. The speed of $M S$ is fixed. The objective of [11], [12] is to maximize the quality data-collection using a constrained-path $M S$. They developed heuristic algorithms for the problem. Addressing a similar problem, Zhang et al. [20] formulated their devised problem as an approximated network-utility maximization problem, and thereafter devised a distributed data-collection method to solve. However, in these works, the time-slot assignment is based on the sensors' current energy and the amount of past data transmitted and the data availabilities that can be sent to the current slot. In our work, to allocate the time-slot for the sensors, we not only consider their current energy, the amount of past data transmitted and current data availabilities but also future data transmission capabilities.

\section{NETWORK MODEL AND PROBLEM FORMULATION}

We consider an EH-WSN as a directed communication graph $G=(R S \cup M S, E)$, as shown in Fig 1. The set $R S=$ $\left\{r s_{1}, r s_{2}, \ldots, r s_{n}\right\}$ denotes stationary deployed sensor nodes with communication radii $\left\{c r_{1}, c r_{2}, \ldots, c r_{n}\right\}$ along a constrained-path $P_{M S}$. The path $P_{M S}$ has fixed path-length $L$. The $M S$ is a mobile-sink used for collecting sensed-data. It moves on the path $P_{M S}$ at speed $V$ for collecting sensed- data from $S N s$ in set $R S$ via single-hop relay. The mobile- sink $M S$ has enough computational power, sufficient energy and memory capacity. In addition, it is well aware about the communication range and the maximum battery capacity of each sensor $r s_{i} \in R S$ beforehand. There exist transmission links in $E$ for $S N s$ in set $R S$ when the $M S$ is within their communication regions. The $M S$ receives data from a sensor $r s_{i} \in R S$ when it passes through the transmission range cri of the sensor $r s_{i}$.

Each sensor $r s_{i}$ has a rechargeable battery with a maximum capacity $C\left(r s_{i}\right)$, to harvest energy. The renewable energy sources can be thermal energy, wind energy or solar energy. Moreover, every sensor is sensing data continuously from the sensing environment, but, we here consider the total amount of data that will be gathered by $M S$ during a complete path- traversal since the same technique can be further exploited to determine data-collection for the next subsequent rounds. 
Further, the trajectory-path $P_{M S}$ is a smooth line segment that can be easily customized for real-application scenarios. In this article, the complete traversal of path $P_{M S}$ by the $M S$ is referred to as a round.

Given the mobile-sink's speed $V$ and the trajectory-path $P_{M S}$, the time duration per round $T_{D G}$ is considered as discrete and is determined as

$$
T_{D G}=\frac{L}{V}
$$

Further, it is slotted into $m=T_{D G} / \tau$ number of time-slots where each time-slot is at-most $\tau$ seconds [21]. Let a set $T_{s}=$ $\left\{t_{1}, t_{2} \ldots t_{m}\right\}$ denote a set of time-slots per

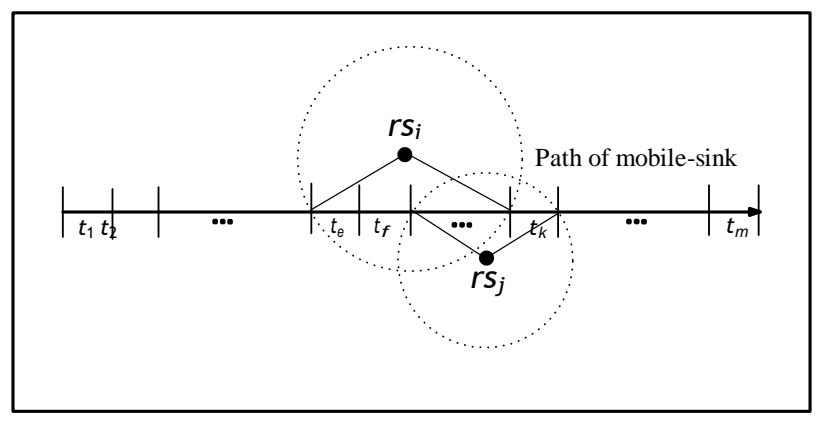

Fig 2. An example of time-slots, on the path, covered by sensors $\mathrm{rs}_{\mathrm{i}}$ and $\mathrm{rs}_{\mathrm{j}}$

The $M S$ broadcasts messages at the start of each time-slot and gets acknowledgment of locations and energy-budgets of the sensors whose communication ranges cover the path $P_{M S}$ partially in that time-slot. In this way, the $M S$ detects the sensors those can send their sensed-data in each time-slot. Further, let $A\left(r s_{i}\right)$ denote a set of succeeding time-slots along the trajectory-path $P_{M S}$. It is noted that, in some time- slots, more than one sensor may transmit their data to the $M S$, as in Fig 2. Therefore, to avoid a collision in each time-slot $t_{j} \in A\left(r s_{i}\right)$, the $M S$ will collect data from only one of them. Therefore, with the aim of maximizing the data delivery contribution of every sensor $r s_{I} \in R S$, we have to allocate time-slots in set $T_{s}$ to every sensor.

\section{A. Energy-harvesting and consumption model}

In this article, the energy of a sensor $r s_{\mathrm{i}} \in R S$ is consumed only in the transmission of data [12]. To support uninterrupted service in each round, a sensor must have an adequate amount of energy for data transmission [4]. We consider that the rate of energy-harvesting of all sensors is lower the rate of their energy-depletion. In addition, they update their energy at the start of every time-slot. Due to dynamic harvesting nature, the energy-harvesting rate is not perfectly deterministic. It is based on the source types and previous harvesting details [9].

Let $B_{i}\left(t_{i}\right)$ be the available energy of a sensor $r s_{i}$ at the start of time-slot $t_{k}$. Let $H_{i}\left(t_{k}-1\right)$ and $Q_{i}\left(t_{k}-1\right)$ denote the amount of energy-harvested and energy-consumed at the $\left(t_{k}-1\right)$ timeslot, where $0 \leq B_{i}\left(t_{k}-1\right) \leq C\left(r s_{i}\right)$.Thus, $B_{i}\left(t_{k}\right)$ is expressed as:

$B_{i}\left(t_{k}\right)=\min \left(B_{i}\left(t_{k-1}\right)+H_{i}\left(t_{k-1}\right)+Q_{i}\left(t_{k-1}\right), C\left(r s_{i}\right)\right.$.

In other words, the harvested energy amount of a sensor should be less than its battery capacity in every time-slot [9]. To achieve perpetual operations, a sensor should not consume more energy than it can deliver data. Therefore, the estimation of energy-budget $\left(t_{j}\right)$ of a sensor $r s_{i}$ at time-slot $t_{j}$ is defined as:

$$
\hat{B_{i}}\left(t_{j}\right)=\min \left\{\sum_{k=t_{j}-q}^{t_{j}-1} \alpha B_{i}\left(t_{j}\right), C\left(r s_{i}\right)\right\},
$$

where $\alpha$ be a constant and $q$ is number of preceding time-slots.

\section{B. Network-utility}

As the energy harvesting rate of a sensor is dynamic, a sensor with sufficient energy harvesting rate may get more chances for delivering its data to the $M S$ within the time duration $T_{D G}$, while another sensor with too low energy may never have any such chances. Consequently, collecting total data from all sensors within time $T_{D G}$ may be biased, which in turn may depict the data-landscape view of the network. Hence, for characterizing the impact of sensed- data collected from each sensor and minimizing the largest difference between the amounts of data transmitted by any two nodes, we present a network-utility function $U($ ). The function $U()$ is non-decreasing and a concave function.

Let $D P_{r s_{i}}$ be the amount of data which is already sent by the sensor $r s_{i}$ to the $M S$ in the past, $D C_{r s_{i}}$ denote the amount of data available to send currently by $r s_{i}$ to the $M S$. And, let $D F_{r s_{i}}$ denote the amount of data which can be sent in the future to the $M S$ for some subsequent time-slots. Let $D_{r s_{i}}$ express as.

$\sqrt{\left(D_{r s_{i}}\right)}=\sqrt{\left(D P_{r s_{i}}+D C_{r s_{i}}+D F_{r s_{i}}\right)}-\sqrt{D P_{r s_{i}}}-\sqrt{D F_{r s_{i}}}$ Then, the network-utility accrued by the sensor $r s_{i}$ will be expressed as:

$$
U\left(D_{r s_{i}}\right)=\sqrt{D}_{r s_{i}}
$$

And, the accumulated sensors' network-utility will be expressed as:

$$
U_{\text {total }}=\sum_{i=1}^{n} \sqrt{D_{r s i}}
$$

which is strictly concave.

\section{Problem definition}

Given an energy-harvesting network $G$ and a set of timeslots $T_{s}=\left\{t_{1}, t_{2} \ldots t_{m}\right\}$ per round in which the $M S$ can collect data, our objective is maximizing the network-utility by assigning the time-slots to each sensor under the following constraints: 
- For a given time-slot $t_{j} \in T_{s}$, a sensor $r s_{i} \in R S$ can send its data to the $M S$ only when the $M S$ is within its communication range.

- A sensor $r s_{i}$ is allowed to transmit data for a set of possible time- slots $A\left(r s_{1}\right) \subset T_{s}$.

- If more than one sensors share the same time-slot, then at-most one of them can deliver its data to the $M S$.

- A sensor cannot consume more energy than its energybudget per time interval.

We refer the stated problem as maximizing quality datacollection $(M Q D C)$ problem.

Theorem 1. The MQDC problem in EH-WSN is an NP-Hard.

Proof: To prove the theorem, we show that the generalized assignment problem (GAP) is a special instance of the $M Q D C$ problem. Let us consider an instance of GAP, where there can be a set of jobs $J=\left\{J_{1}, J_{2}, \ldots, J_{m}\right\}$ and a set of agents $A=\left\{A_{1}, A_{2}, \ldots, A_{n}\right\}$ with resource capacities $B=\left\{b_{1}, b_{2}, \ldots, b_{n}\right\}$, where for each job $J_{k} \in J$ and an agent $A_{j} \in A, b_{j k}$ is the amount of resources used by $A_{j}$ to perform job $J_{k}$ and $p_{j k}$ be the profit achieved if job $J_{k}$ is assigned to $A_{j}$, the goal is to assign jobs in set $J$ to the agents in set $A$ such that total earned profit is maximized, subjected to the capacity limit of each agent.

We formulate a corresponding $M Q D C$ problem by assuming that every sensor is able to deliver its sensed-data in all time-slots of set $T_{s}$, where jobs are modeled as time-slots, sensors and their energy-budgets are modeled as agents and their resource capacities. Furthermore, for a time-slot $t_{k}$ and a sensor $r s_{j}$, the energy-depletion of the sensor $r s_{j}$ is $b_{j k}$, if the sensor $r s_{j}$ is allowed to transfer $p_{j k}$ amount of data within a time-slot $t_{k}$. Therefore, the $M Q D C$ problem is reducible into GAP; hence, it is NP-hard.

\section{PROPOSED SOLUTION}

So, each sensor can deliver data only when the time-slots are assigned to it. In this algorithm, at every iteration, we examine a single time-slot for assigning it to at-most sensor. This process repeats until all the time-slots have been assigned to sensors. Later, we will describe in detail how to assign a time-slot among all given time-slots. For a given time-slot $t_{j}$, let $N\left(t_{j}\right)=\left\{r s_{i} \mid t_{j} \in A\left(r s_{i}\right)\right\}$ denote a set of candidate sensors that can transmit their data to the time-slot $t_{j}$. So, if $N(j)=\Phi$, implies that no any sensor can send its data at time-slot $t_{j}$. This may occur either because all sensors can't communicate with the $M S$ or because they don't have sufficient energy-budgets to transmit data. Otherwise, the time- slot $t_{j}$ is assigned to sensor $r s_{i} \in N\left(t_{j}\right)$. The process for selecting the sensor $r s_{i}$ among all sensors in set $N\left(t_{j}\right)$ as follows

As stated earlier, $U\left(D_{r s_{i}}\right)=\sqrt{D}_{r s_{i}}$ which is nothing but equals to

$$
\sqrt{\left(D P_{r s_{i}}+D C_{r s_{i}}+D F_{r s_{i}}\right)}-\sqrt{D P_{r s_{i}}}-\sqrt{D F_{r s_{i}}}
$$

is the utility gain of the sensor $r s_{i}$ by allocating time-slot $t_{j}$ to it for transmitting its data. For maximizing the network-utility, we assign time-slot $t_{j}$ to the sensor which has maximum utility gain among all sensors in set $N\left(t_{j}\right)$.

The summarized form of MQDCPMS algorithm is presented as follows:

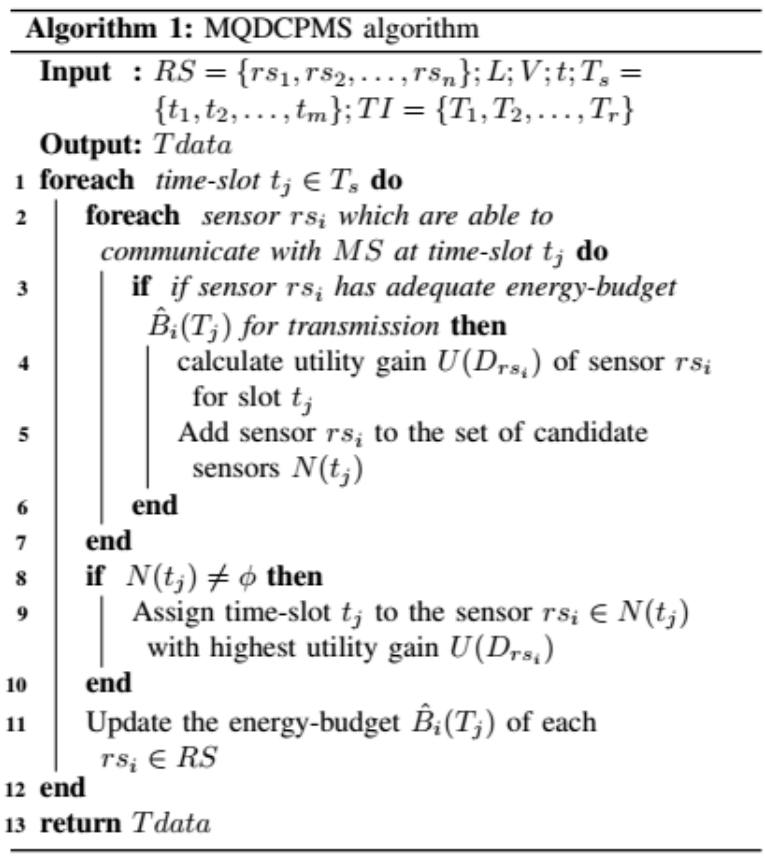

\section{A. Complexity analysis}

Theorem 2. The Run-time complexity of MQDCPMS algorithm is $O(m n)$.

Proof. In MQDCPMS algorithm, at each iteration, a single time-slot $t_{j}$ will be assigned, which takes $O(n)$ time com- plexity due to the creation of a set $N(j)$ and determining a potential sensor in $N(j)$. The size $\mathrm{m}$ of the time-slots in set $T_{s}$ determines the total number of iterations of MQDCPMS algorithm. Hence, the overall run-time complexity of MQDCPMS is $O(m n)$.

\section{PERFORMANCE EVALUATION}

We here evaluate the quality data-collection performance of the proposed algorithm MQDCPMS by comparing with Cschedule algorithm [12] in terms of network-utility throughput. The simulation experiments of the algorithm MQDCPMS are implemented in MATLAB. We analyze the effect of change in network size and the change in MS speed on the network- utility metric.

\section{A. Experimental setup}

For the simulation experiments, we have considered an EH-WSN that consists of 100-600 sensors (SNs) randomly distributed nearby the constrained-path of $M S$. The length of the constrained -path is $1800 \mathrm{~m}$. 
The locations of all SNs are at-most 190m away from the $M S$ path. The common transmission range of all sensors is set to $200 \mathrm{~m}$. The various speeds of $M S$ are considered in analyzing the network-utility performance of the network. A solar panel with dimension $10 \mathrm{~mm} \times 10 \mathrm{~mm}$ is used for powering each sensor. The battery-capacity of each sensor is $10,000 \mathrm{~J}$ (Joules). The data availability of a sensor is a random value within $250-1000 \mathrm{~Kb}$. In this work, the energy transmission cost is set to $300 \mathrm{~mJ} / \mathrm{sec}$.

The different data-transmission rates of sensors are defined as follows. In the range of $0-20 m$, the data- transmission rate is $250 \mathrm{Kbps}$, in the range of $20-50 \mathrm{~m}$, it is $19.2 \mathrm{Kbps}$, in the range of $50-120 \mathrm{~m}$, it is $9.6 \mathrm{Kbps}$, and in the range of $120-200 \mathrm{~m}$, it is $4.8 \mathrm{Kbps}$. The duration of each time-slot is set to $1 s$.

\section{B. Impact of network size on Network-utility}

We evaluate the quality data-collection performance of the MQDCPMS algorithm and compare it with the algorithm Cschedule [12] in terms of network-utility throughput. As shown in Fig 3, For the $M S$ speed at $5 \mathrm{~m} / \mathrm{sec}$, the network-utility of MQDCPMS and C-schedule increases with the increment in the network size. In addition, the MQDCPMS algorithm has more network-utility than the $\mathrm{C}$-schedule algorithm. This is because the MQDCPMS uses comparatively better utility function compared to $\mathrm{C}$-schedule. The MQDCPMS not only uses past and current data availability, but also future data for minimizing the largest difference between the amounts of data transmitted by the two nodes. We here evaluate the impact of change in $M S$ speed on network-utility for MQDCPMS algorithm. Fig. 4 presents that with the increments in $M S$ speed, the network-utility decreases. This is so because, at low speed, a number of time- slots become available for the sensors. However, this will cause a longer delay on data-collection

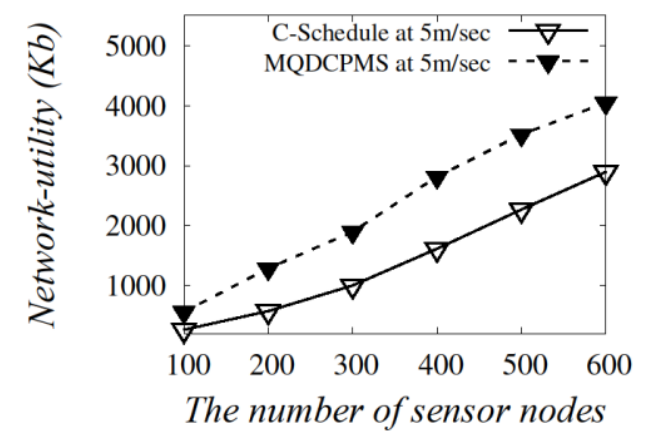

Fig. 3. Network-utility for $M S$ speed $5 \mathrm{~m} / \mathrm{sec}$

\section{Impact of MS speed on Network-utility}

We here evaluate the impact of change in $M S$ speed on network-utility for MQDCPMS algorithm. Fig. 4 presents that with the increments in $M S$ speed, the network-utility decreases. This is so because, at low speed, a number of timeslots become available for the sensors. However, this will cause a longer delay on data-collection.

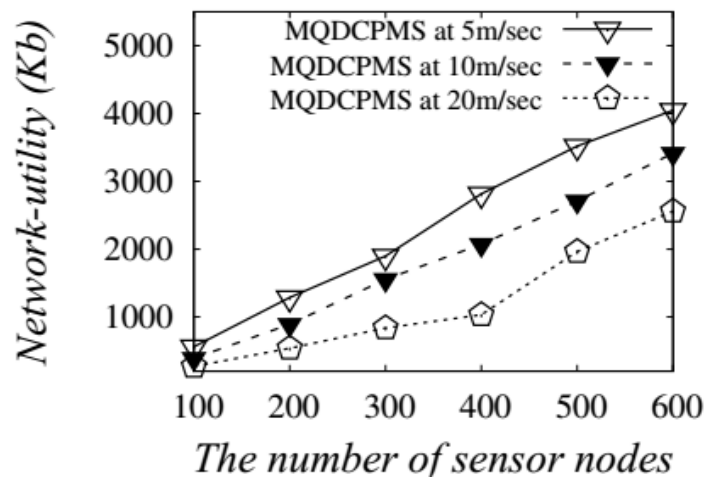

Fig. 4. Network-utility for distinct $M S$ speeds

\section{CONCLUSION.}

We studied our proposed $M Q D C$ problem in an EH-WSN with a fixed-path mobile-sink $M S$. To prove NP-hardness, we converted the problem $M Q D C$ into the GAP problem. Then, we proposed MQDCPMS algorithm to solve this. In MQDCPMS, we have considered different parameters such as current energy level, historical amount of data transmitted, the current amount of data to be transmitted and future amount of data that can be transmitted with the utility function for the slot time allocation. The experimental results are the average of 25 distinct topologies of same size network. The results reveal that the algorithm MQDCPMS is more effective than the existing one. As future work, determining a trajectory-path for $M S$ in large-scale EH-WSNs supporting single-hop relay through theoretical as well as experimental analysis can be considered.

\section{ACKNOWLDGEMENT}

This work is supported by the Science I\& Engineering Research Board, DST, Govt. of India [Grant numbers: ECR/2016/001035];

\section{REFERENCES}

1. V. P. Gil Jiménez and M. J. Ferna ńndez-Getino Garc' 1a, "Simple design of wireless sensor networks for traffic jams avoidance," Journal of Sensors, vol. 2015.

2. M. A'. Serna, R. Casado, A. Bermu'dez, N. Pereira, and S. Tennina, "Distributed forest fire monitoring using wireless sensor networks," International Journal of Distributed Sensor Networks, vol. 11, no. 10, p. 964564, 2015.

3. K. Z. Panatik, K. Kamardin, S. A. Shariff, S. S. Yuhaniz, N. A. Ahmad,O M. Yusop, and S. Ismail, "Energy harvesting in wireless sensor networks: A survey," in Telecommunication Technologies (ISTT), 2016 IEEE 3rd International Symposium on. IEEE, 2016, pp. 53-58.

4. C. Renner, S. Unterschu"tz, V. Turau, and K. Ro"mer, "Perpetual data collection with energy-harvesting sensor networks," ACM Transactions on Sensor Networks (TOSN), vol. 11, no. 1, p. 12, 2014.

5. T. Noushad and K. Sebastian, "Review on energy harvesting and data collection," in IOP Conference Series: Materials Science and Engineer- ing, vol. 396, no. 1. IOP Publishing, 2018, p. 012025. 


\section{Maximizing Quality Data-collection in Mobile-sink Based Energy-harvesting Sensor Networks}

6. M. Prauzek, J. Konecny, M. Borova, K. Janosova, J. Hlavica, and Musilek, "Energy harvesting sources, storage devices and system topologies for environmental wireless sensor networks: A review," Sensors, vol. 18, no. 8, p. 2446,2018

7. K. Patil, K. De Turck, and D. Fiems, "Optimal data collection in hybrid energy-harvesting sensor networks," in International Conference on Analytical and Stochastic Modeling Techniques and Applications. Springer, 2016, pp. 239-252.

8. Y. Gu, F. Ren, Y. Ji, and J. Li, "The evolution of sink mobility man- gement in wireless sensor networks: A survey," IEEE Communications Surveys \& Tutorials, vol. 18, no. 1, pp. 507-524, 2016.

9. A. Mehrabi and K. Kim, "Maximizing data collection throughput on a path in energy harvesting sensor networks using a mobile sink," IEEE Transactions on Mobile Computing, no. 3, pp. 690-704, 2016.

10. A. Mehrabi, "Optimal speed allocation in sink-based energy harvesting wireless sensor networks," in 2018 IEEE 32nd International Conference on Advanced Information Networking and Applications (AINA). IEEE, 2018, pp. 128-134.

11. X. Xu, W. Liang, and T. Wark, "Data quality maximization in sensor networks with a mobile sink," in 2011 International Conference on Distributed Computing in Sensor Systems and Workshops (DCOSS). IEEE, 2011, pp. 1-8.

12. X. Ren and W. Liang, "The use of a mobile sink for quality data collec- tion in energy harvesting sensor networks," in Wireless Communications and Networking Conference (WCNC), 2013 IEEE. IEEE, 2013, pp. 1145-1150.

13. C. Wang, S. Guo, and Y. Yang, "Energy-efficient mobile data collection in energy-harvesting wireless sensor networks," in 2014 20th IEEE International Conference on Parallel and Distributed Systems (ICPADS). IEEE, 2014, pp. 55-62.

14. Z. Zhou, C. Du, L. Shu, G. Hancke, J. Niu, and H. Ning, "An energybalanced heuristic for mobile sink scheduling in hybrid wsns," IEEE ransactions on Industrial Informatics, vol. 12, no. 1, pp. 28-40, 2016.

15. L. Fu, L. He, P. Cheng, Y. Gu, J. Pan, and J. Chen, "Esync: Energy synchronized mobile charging in rechargeable wireless sensor networks," IEEE Transactions on Vehicular Technology, vol. 65, no. 9, pp. 74157431,2016

16. X. Ren, W. Liang, and W. Xu, "Use of a mobile sink for maximizing data collection in energy harvesting sensor networks," in Parallel Processing (ICPP), 2013 42nd International Conference on. IEEE, 2013, pp. 439-448.

17. X. Ren and W. Liang, "Delay-tolerant data gathering in energy harvest- ing sensor networks with a mobile sink," in Global Communications Conference (GLOBECOM), 2012 IEEE. IEEE, 2012, pp. 93-99.

18. A. Mehrabi and K. Kim, "Optimal transmission period for improved sink-based data collection in energy harvesting wireless sensor net- works," in 2016 IEEE International Conference on Communications (ICC). IEEE, 2016, pp. 1-6.

19. K. Li, C. Yuen, B. Kusy, R. Jurdak, A. Ignatovic, S. Kanhere, and S. K. Jha, "Fair scheduling for data collection in mobile sensor networks with energy harvesting," IEEE Transactions on Mobile Computing, 2018.

20. Y. Zhang, S. He, and J. Chen, "Near optimal data gathering in recharge- able sensor networks with a mobile sink," IEEE Transactions on Mobile Computing, vol. 16, no. 6, pp. 1718-1729, 2017.

21. L. Lin, N. B. Shroff, and R. Srikant, "Asymptotically optimal energy- aware routing for multihop wireless networks with renewable energy sources," IEEE/ACM Transactions on Networking (TON), vol. 15, no. 5, pp. 1021-1034, 2007.

\section{AUTHORS PROFILE}

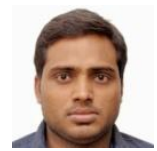

Naween Kumar received the bachelor and master degrees in computer application from Indira Gandhi National Open University in 2007 and 2010, respectively. $\mathrm{He}$ is currently working toward the $\mathrm{PhD}$ degree in the Dept of CSE, National Institute of Technology Patna, India. His research interests include designing energy efficient data collection protocol and mobility based data collection in Wireless sensor network and Energy-harvesting sensor network.

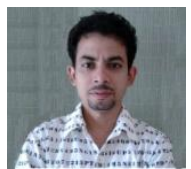

Dr. Dinesh Dash received a Master of Technology in Computer Science and Engineering from University of Calcutta, India in 2004. From 2004 to 2007 he worked as a Lecturer under West Bengal University of Technology, India. He was awarded Ph.D. in 2013 from Indian Institute of Technology Kharagpur, India. His $\mathrm{PhD}$ research topic was on the Coverage Problems in Wireless Sensor Network. He worked as senior research associate from 2013 to 2014 at Infosys Limited, India. From
2013 to 2014 he worked as Assistant Professor at Tezpur University, Assam, India. Since Dec 2014, he is working as an Assistant Professor in the Dept of CSE, National Institute of Technology Patna, India. His current work focuses on the sensor network coverage problem, data gathering problem, design of fault tolerant system in the mobile AdHoc Network. 